Cinémas

Revue d'études cinématographiques

Journal of Film Studies

\title{
Le tapis incertain. Métamorphose et hors-champ dans Cat People
}

\section{Vicente Sánchez-Biosca}

Volume 5, numéro 3, printemps 1995

Cinélekta 1

URI : https://id.erudit.org/iderudit/1001145ar

DOI : https://doi.org/10.7202/1001145ar

Aller au sommaire du numéro

Éditeur(s)

Cinémas

ISSN

1181-6945 (imprimé)

1705-6500 (numérique)

Découvrir la revue

Citer cet article

Sánchez-Biosca, V. (1995). Le tapis incertain. Métamorphose et hors-champ dans Cat People. Cinémas, 5(3), 29-44. https://doi.org/10.7202/1001145ar
Résumé de l'article

Cat People (Lewton-Tourneur, 1942) est traversé par une poétique de l'incertain. C'est essentiellement ce qui l'oppose à la fois à la tradition gothique des films fantastiques des années quarante et aux films d'épouvante actuels. La subtilité des relations entre le vu et le non-vu, le rôle primordial accordé au silence et au non-dit, toute une rhétorique de la suggestion et de l'absence font de ce film un point de clivage dans l'histoire du fantastique au cinéma. 


\section{Le tapis incertain. Métamorphose et hors-champ dans Cat People}

\section{Vicente Sánchez-Biosca}

\section{RÉSUMÉ}

Cat People (Lewton-Tourneur, 1942) est traversé par une poétique de l'incertain. C'est essentiellement ce qui l'oppose à la fois à la tradition gothique des films fantastiques des années quarante et aux films d'épouvante actuels. La subtilité des relations entre le vu et le non-vu, le rôle primordial accordé au silence et au nondit, toute une rhétorique de la suggestion et de l'absence font de ce film un point de clivage dans l'histoire du fantastique au cinéma.

\section{ABSTRACT}

Cat People (Lewton-Tourneur, 1942) is traversed by a poetic of the uncertain. This is primarily what sets it apart from the gothic tradition of the fantastic films of the 1940s and also from today's horror films. The subtlety of relationships between the seen and the unseen, the primordial role played by silence and the unsaid, and an entire rhetoric of suggestion and absence make this film a turning point in the history of fantastic cinema.

En juin 1942, Peter Rathvon devint le nouveau président au sein de l'instable RKO, tandis que Charles Koerner prit la relève de la production. Encore une fois, la politique du studio fut bouleversée et les préférences de Koerner se firent bientôt sentir. Au lieu de parier sur de grandes et prestigieuses productions de série $\mathrm{A}$, il orienta le marché dans la direction des films peu coûteux, c'est-à-dire des films d'exploitation. Ce fut le début de 
la période la plus prospère de la $\mathrm{RKO}$, laquelle dura quatre ans, et ce n'est pas par hasard si sa fin coïncide avec le décès de Koerner lui-même, le 2 février 1946, et sa substitution par Dore Schary, scénariste et producteur provenant de la MGM. Celui-ci inaugure une politique tout à fait opposée à celle de son prédécesseur. Il entreprend des productions audacieuses qui vont, malgré leur prestige, affecter sérieusement le bilan des bénéfices. C'est sous le règne de Koerner que Val Lewton, un romancier ayant travaillé comme story editor pour David O. Selznick entre 1933 et 1942, est invité à former une équipe de production attachée au tournage des films de série B. L’on ne saurait assez insister sur l'opportunité historique d'une telle rencontre. La situation économique du studio, la politique de production de Koerner - un producteur capable de mettre sur pied un groupe bien serré d'artistes (en somme un producteur capable de se doter d'une direction esthétique) - et, on y reviendra, le travail d'ensemble au sein de cette équipe ont contribué à favoriser le travail de Lewton et à façonner la production de cette époque à la RKO.

C'est ainsi que l'unité de production de Val Lewton lance, entre 1942 et 1945 , onze films destinés à constituer la partie complémentaire des double bills, ces modes généralisés de distribution en bloc qui s'imposaient à ce moment. Les conditions de production, de distribution et d'exploitation de ces films étaient fixées d'avance: tournage rapide en studio ne dépassant jamais les quatre semaines, durée maximale de 75 minutes, budget inférieur aux 150000 \$. Il n'y a aucun doute, quel que fut le succès rétrospectif de ces films auprès des historiens, ils furent conçus, produits, distribués et exploités à titre de films de série B '. Comme Lea Jacobs l'a souligné récemment: "The horror films of Val Lewton, for example, were consistently screened as Bs, opening at the small Rialto Theatre in New York and quickly relegated to split double and triple bills outside the New York metropolitan area " (p. 13). Il faudrait ajouter à cet anonymat l'indifférence de la réception critique. À quelques exceptions près, "[...] these films were dismissed by the critical establishment as well, despite the fact that critics Manny Farber and James Agee championed Lewton's work» (p. 13). Quoi qu'il en 
soit, à l'intérieur des limites imposées par les conditions de production, il semblerait, selon les déclarations des artisans, que les magnats de RKO ne se soient jamais mêlés aux détails de la production ni avoir posé des restrictions additionnelles à la méthode adoptée par Lewton et par son groupe. Ainsi, une sorte de travail collectif, peu fréquent par ailleurs dans le milieu hollywoodien, réussit à s'imposer.

Il est partout admis maintenant (et c'est presque un mythe) que l'équipe Lewton était en possession d'une unité stylistique toute reconnaissable, à tel point que certains auteurs en sont venus à postuler la lecture d'un seul macrotexte. C'est peut-être aller trop loin. Néanmoins, il vaut la peine de "re-situer", à l'intérieur de ce corpus, les trois premiers films, ceux qui furent mis en scène par Jacques Tourneur: Cat People (La Féline, première : décembre 1942), I Walked with a Zombie (Vaudou, première: avril 1943) et The Leopard Man (L'Homme léopard, première: mai 1943). Les noms des artisans, qui se répètent (Val Lewton, le metteur en scène Jacques Tourneur, le monteur Mark Robson, le musicien Roy Webb, le directeur artistique Albert D'Agostino), les considérations connues sur l'équipe Lewton et la continuité dans le temps de ces trois productions suggèrent une unité effective ${ }^{2}$.

Or, les particularités de ces trois films sont loin d'être épuisées par leur condition de films "B ". En réalité, s'ils ont été élevés à la dignité de films cultes, c'est surtout à cause de l'expression qu'ils donnent à la notion de "fantastique". Si nous envisageons la dimension diachronique, nous sommes frappés par le changement radical que ces films produisirent dans la tradition des films d'horreur de l'Universal des années trente, tradition encore dominante au début des années quarante ${ }^{3}$. La subtilité de la relation entre monstration et dissimulation, le rôle axial des silences, le règne de la suggestion et le déploiement d'une rhétorique du non-dit... : voilà autant de traits qui sont devenus des lieux communs lorsqu'on parle des productions Lewton. Mais aussi, rien de plus étranger aux monstres d'inspiration gothique, qui pullulent pendant la décennie précédente dans le cinéma de l'Universal, que ces figures fantastiques se dissimulant dans les films du duo Lewton-Tourneur. Par conséquent, il n'est pas 
étonnant qu'on ait eu recours à la théorie de Todorov sur le fantastique pour définir ces films. Tom Gunning, par exemple, lorsqu'il analyse Cat People, s'appuie sur l'idée todorovienne de "discours figuratif" ainsi que sa correspondance avec la suspension du sens ${ }^{4}$.

Par conséquent, la modernité des films Lewton semble être liée au fantastique si l'on conçoit celui-ci comme une poétique de l'incertain qui s'opposerait au renouveau gothique du début des années trente. Evelyne Lowins a mis en rapport l'imagerie des films Lewton avec l'inquiétante étrangeté ou Unheimlich freudienne. Fracture du sens, vacillation interprétative, assujettissement du point de vue du spectateur à l'incertitude et à l'émergence du passé refoulé sur le présent... Faudrait-il voir ici une transgression de la mise en scène transparente hollywoodienne? Un retournement des formes classiques qui en dévoile le point de rupture, comme le propose Bernard Eisenschitz ${ }^{5}$ ? Ou bien, serions-nous tentés d'y découvrir une expression de la nostalgie d'un langage primordial et à valeur magique, comme le suggère Tom Gunning (p. 35), en rappelant les conceptions linguistiques du romantisme allemand? Ou encore, suivant la radicalité de Mary Ann Doane à propos de Cat People, y verrat-on une matérialisation des "manques théoriques" de la psychanalyse lorsqu'elle a affaire à la sexualité féminine (p. 49-52) ?

Cependant, on ne saurait se tromper à propos de la supposée modernité des films de Val Lewton. Si nous les avons qualifiés de modernes en raison de leur mise en place du fantastique et par opposition aux monstres gothiques, nous sommes forcés, en revanche, de les considérer comme une expression bien archaïque par rapport au cinéma d'horreur actuel. En effet, les jeux d'ombres, les dark patches ou horror spots, tels que Lewton luimême en parle et en esquisse une théorie ${ }^{6}$, le travail des absences visuelles et sonores ainsi que l'atmosphère envoûtante qui opère par suggestion ne sont plus depuis longtemps des traits reconnaissables du cinéma d'épouvante. Ce qui détermine cette différence est un fait de représentation qu'il vaudrait mieux énoncer brutalement: après Psycho (Hitchcock, 1960) ou, pour mieux dire, à la suite de la séquence du meurtre dans la douche, le cinéma de terreur et son spectateur ont refusé de retirer le 
regard du lieu fascinant, et en même temps insupportable, où Psycho l'avait attiré, le lieu tragique où un corps féminin était sauvagement déchiré. Il s'agira désormais d'offrir à l'œil les moments de jouissance, tout spécialement ceux qui ont affaire à des images du corps morcelé: aux métamorphoses, aux exercices de violence, aux explosions de la chair, aux irruptions du cadavre en tant que support chimique du corps au-delà même de sa condition vivante ${ }^{7}$... Et pourtant, Psycho, malgré son geste, n'allait pas aussi loin, il n'offrait à l'œil qu'une métaphore précaire par le biais du montage. Celui-ci, en morcelant à son tour la scène et par conséquent le corps lui-même, mais en recouvrant en même temps la violence de la déchirure provoquée par le couteau, réussissait à "spectaculariser" la violence, tout en nous préservant de sa perception brute ${ }^{8}$. Voilà le paradoxe d'un film comme Psycho. Par contre, le cinéma d'horreur qui le suit a fait son choix, il préfere l'utilisation de la mise en scène la plus neutre possible, il préfere les plans moyens et refuse le montage, autant de stratégies qui ont surpris les historiens. Oserions-nous dire que ce qui était une métaphore du montage dans Psycho morceler un corps, le couper par un double instrument - a ouvert la voie aux effets spéciaux dont la réalisation a lieu dans le champ'? L'offre à l'œil de l'objet, pour sa dévoration, est la manifestation de la pulsion scopique telle qu'elle se montre aujourd'hui. Voici les traits décisifs de la terreur actuelle qui, du même coup, rendent désuets les films de Lewton-Tourneur, précisément à cause de leur travail de mise en scène. Récapitulons: modernité vis-à-vis du gothique des années trente, archaïsme par rapport au cinéma de terreur actuel. Faut-il insister? Sur la scène historique, les films de Lewton se révèlent un point de clivage, voire des textes limites.

\section{Le germe de l'équipe Lewton}

Cat People. Voilà l'expression inaugurale de l'équipe Lewton et, à coup sûr, le film qui cristallisa son sens du fantastique et des craintes quotidiennes. D'après Telotte, le scénariste DeWitt Bodeen aurait rédigé un brouillon du scénario sous la forme d'une nouvelle ouverte à l'incorporation de nouvelles scènes. Ce fut le groupe de production, réuni en entier, qui analysa et révisa 
minutieusement l'histoire pour offrir à Lewton un découpage élaboré qu'il était censé corriger personnellement. Le film fut tourné rapidement, comme cela deviendra l'habitude par la suite, entre le 28 juillet et le 21 août 1942 dans le studio de la RKO. L'histoire dont il s'agit est bien connue. Irena Dubrovna (Simone Simon), jeune Serbe habitant New York, est hantée par la légende de son village natal qui prédit sa conversion en panthère lors d'un contact sexuel avec un homme. Elle vit dans une atmosphère envoûtante, pleine de représentations félines (des tapis décorant les murs jusqu'aux statuettes dans le salon en passant par les esquisses qu'elle peint compulsivement). Est-ce par hasard qu'elle a choisi de vivre près du zoo où se font entendre les rugissements de la panthère dont Irena affirme qu'elle "crie comme une femme" ? Néanmoins, non seulement la vue et l'ouïe du personnage sont sollicités, mais de plus l'odeur de son appartement (on sait bien que la panthère est reconnaissable à son odeur) est liée aux représentations des peurs primordiales dans lesquelles Irena a décidé de vivre. Autant de signes, donc, qui font planer des connotations félines sur une quotidienneté anodine.

Or, le noyau de Cat People contient une violence au corps humain (c'est-à-dire la métamorphose) et dans la même mesure une violence au langage en tant que support des structures anthropologiques qui garantissent la distinction fondatrice entre l'animal et l'humain. Il serait insuffisant de repérer ici une vacillation d'interprétation; il nous faut plutôt y découvrir un problème sous-jacent de représentation. Un problème qui pose quelques questions, notamment les suivantes: en ce qui concerne la métamorphose, clé de voûte du fantastique, qu'est-ce qui est confié au récit? Qu'est-ce qui est donné à l'œil ? Selon que la réponse se déplace de l'un ou de l'autre coté, nous y distinguerons une de ces deux logiques différentes, voire opposées: métaphore d'un savoir en circulation dans le premier cas; pulsion scopique, plénitude du regard, par ailleurs mythique, impossible, dans le second. Seule une analyse de la mise en scène pourrait nous fournir la clé d'interprétation.

C'est déjà un lieu commun de constater que le développement du film présente une "corporéisation" progressive de la panthère. Quatre fois, elle plane près de la scène: si la première 
fois, lors de la célèbre poursuite près de Central Park (voir l'analyse qui suit), elle reste suspendue hors du cadre, la deuxième fois, il nous est permis de percevoir confusément son ombre autour d'une piscine et d'entendre ses terribles rugissements ${ }^{10}$. La troisième apparition la représente en corps à l'intérieur du bureau et dans un espace pourtant étrangement découpé par le jeu du champ-contrechamp ". Au cours de la quatrième et dernière irruption de la panthère, lors de l'agression finale contre le psychiatre et séducteur Dr. Judd, la visualisation sera à nouveau déviée et le spectateur ne percevra que de nombreuses métonymies de sa présence. Revenons, nonobstant, à notre développement. En tant que point d'origine de cette progression, la première métamorphose constitue le moment le plus décisif du film. Une fois le système connotatif bâti avec tant de soin, la contrainte d'une lecture littérale (et non pas allégorique) de la transformation d'Irena anéantirait le moindre doute par la suite. Voilà la raison pour laquelle, au lieu de se donner à l'œil, la première métamorphose de Cat People mobilise le registre de la métaphore. Examinons de plus près, alors - bien que de façon forcément succincte - , cette séquence qui comporte 36 plans et qui nous force, on y reviendra, à une lecture en surimpression de l'implication statique (composition plastique) et de la diégèse (montage).

Un soir, après une dispute conjugale, Oliver (Kent Smith) se rend à son bureau. Lorsque Irena lui téléphone, elle entend la voix de celle qu'elle considère comme sa rivale, Alice (Jane Randolph). Elle est alors subitement saisie de jalousie et se rend, elle aussi, près du bureau où elle retrouve, dans un café au coin d'une rue, Oliver, soucieux, attablé avec Alice. Irena les observe, sans perdre un seul détail, à travers le rideau d'une fenêtre qui donne sur l'intérieur. Une fois dans la rue, le couple se sépare. En pleine nuit, Irena se met alors à la poursuite d'Alice, poursuite qui va aboutir à la métamorphose. Mais, revenons sur nos pas et suivons de plus près cette séquence.

"Stormy weather", dit Alice lorsqu'elle constate l'attitude préoccupée de son camarade Oliver. À ce moment, par une sorte de logique métonymique, l'effet pour la cause, un fondu enchaîné nous transporte dans un autre plan et de composition fort bizarre. Du côté droit du cadre, Irena s'avance vers la 
caméra. Il est à noter que ce mouvement-ci de l'acteur brise la symétrie du plan précédent ${ }^{12}$ (photogramme 1 ); mais aussi, si nous observons attentivement, nous ne tarderons pas à nous apercevoir que l'image est réfléchie dans un miroir, ce qui signifie qu'il s'agit d'une image virtuelle. Ajoutons à ceci l'implication connotée par le fondu enchaîné et l'on aura l'impression qu'Irena s'élance vers l'espace précédent. En somme, nous avons affaire à une construction surprenante: le point de vue figuratif, le fondu enchaîné, la centralité de la figure noire surgissant de l'ombre, la difficulté à localiser dans l'espace les positions relatives des personnages sont à la base d'une désorientation momentanée du spectateur qui suggère l'idée de guet, dont il s'agit en réalité.

C'est peut-être cette épaisseur du statique au détriment du diégétique qui oblige les plans suivants à recomposer l'articulation des deux espaces en faisant que l'un d'eux (le café) dépende du point de vue qui s'ancre dans l'autre (le regard d'Irena à travers le rideau).

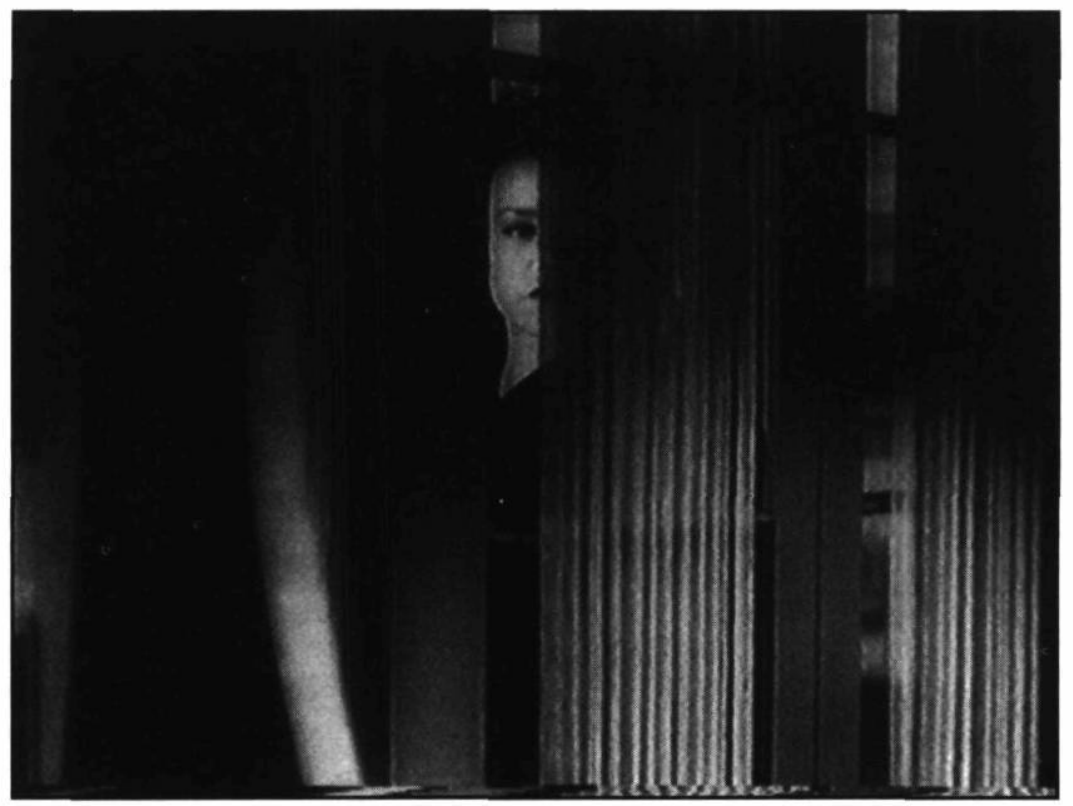

Cette réorganisation du visible ne sera nullement durable, elle va bientôt se fracturer par la présence d'une nouvelle incertitude spatiale se produisant lorsque le couple sort du café. L'emplacement de la caméra est identique au plan 2. En fuyant l'espace virtuel, 
l'image d'Irena se dédouble. Après s'être cachée, Irena apparaît au milieu d'un décor surchargé: des fleurs qui connotent et l'odeur et son aspect sauvage, le miroir qui a marqué la double entrée et sortie du champ, l'obscurité régnante dans le plan... Recréation métaphorique qui contraste avec la neutralité de l'autre espace complètement et étrangement hétérogène par rapport à celui-ci. Opposition bien évidente: un espace prêt à se diégétiser (le café), de décor neutre, figé rétroactivement à un point de vue narratif; un autre espace, incertain, surchargé, métaphorique.

Voici la clé d'une séquence qui nous oblige à lire autant en extension qu'en intention; un double décryptage, donc, qui implique non seulement une logique métonymique, se déplaçant de cause en effet, mais aussi une autre logique métaphorique qui semble arrêter le progrès du récit afin de combler la signification d'une manière statique, iconographique. Ce que la séquence tentera de développer, c'est la projection de l'axe métaphorique sur la diégèse : le guet, en réalité, deviendra la menace, voire la poursuite.

À la fin du plan 9, on assiste à la séparation d'Alice et d'Oliver. Un champ vide résulte d'une double sortie du champ des personnages (la femme par le côté gauche, l'homme par la droite).

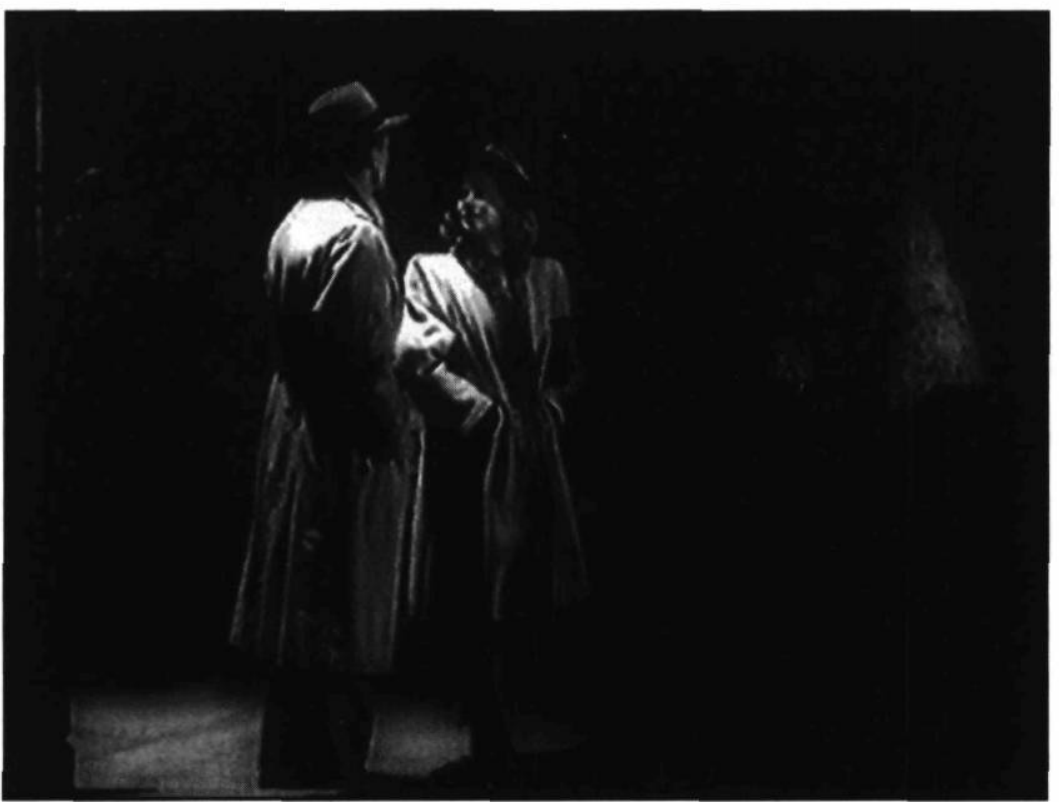


Le plan suivant insiste sur un phénomène que nous avons déjà souligné auparavant. Un fondu enchaîné nous présente Irena avançant vers la caméra. Lisons-le figurativement par rapport au cadre final du plan 9: sur la fracture ouverte au centre du cadre par la sortie des deux personnages, Irena s'élance à nouveau, en projetant la ligne de profondeur sur l'horizontale, mais surtout en fonçant vers le spectateur. Insistance sur la frontalité de l'avancement d'Irena et sur la ligne de profondeur sur laquelle elle se déplace, fort redondante d'ailleurs si l'on tient compte du fait que ce personnage se déplace aussi frontalement au plan 14 tandis qu'Alice le fait latéralement dans le plan suivant.

Nous voilà maintenant au cœur même de la poursuite, dans un décor austère censé représenter les alentours de Central Park. Nous assistons alors à cette construction symétrique :

Plan 15 (latéral) : Alice traverse le champ en plan américain de gauche à droite. Champ vide. Irena traverse le champ dans la même direction.

Plan 16: Travelling en plongée sur les pieds d'Alice. Plan 17: Travelling en plongée sur les pieds d'Irena. Plan. 18: Comme 15. Alice traverse le champ. Champ vide...

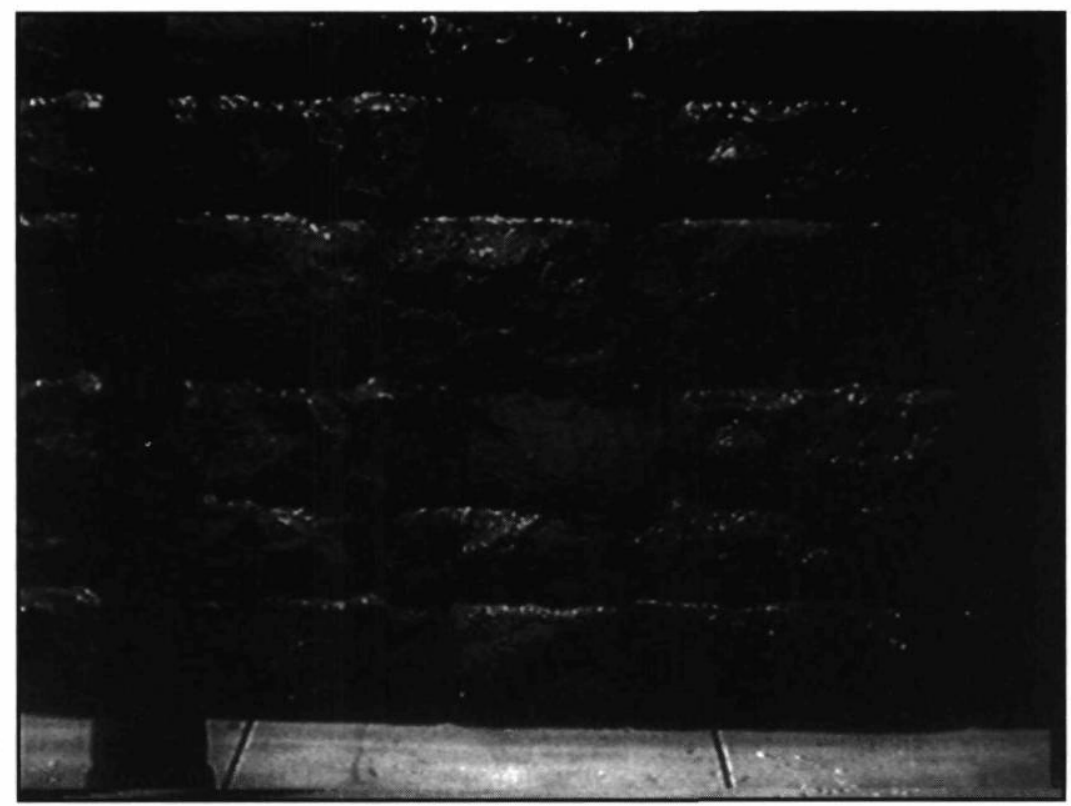


Le plan 15 a pour but de nous offrir, pour la première fois dans la séquence, une définition des coordonnées spatiales (position relative des deux personnages, qui partagent, bien qu’à des moments différents, le même espace), temporelles (bien que sans la dramatisation du montage) et de l'action (celle d'un personnage est en fonction de l'autre). Voilà ce qui est étonnant par rapport à l'indécision des plans précédents. Les plans 16 et 17 introduisent un montage alterné en même temps qu'ils soulignent l'effet sonore (les pas qui avancent à des rythmes divers). C'est lorsqu'on est arrivé au point le plus précis spatialement et temporellement que la séquence suspend radicalement toute orientation et dissout aussitôt les effets qu'elle avait bâtis avec tant de soin. Rupture spatiale, puisque l'image d'Irena n'apparaît pas là où nous l'attendions; rupture temporelle, puisque la durée s'efface dans l'indéfinition. Qu'y a-t-il au lieu du poursuivant? Un vide ou, plus exactement, un double vide, visuel et sonore. Ni le corps n'est visible ni les pas ne sont audibles. Retenons ceci que le moment de la métamorphose, l'instant où l'humain s'évanouit, ne donne pas lieu à l'irruption d'une figuration de l'animal ni de la transformation elle-même (formes composites de l'humain et de l'animal) mais, par contre, qu'elle est incarnée par un double vide que la mise en scène s'efforce d'accentuer. En effet, les plans 19 à 24 (où un autobus fait irruption sur la bande-image et sur la bande-son sous la forme équivoque d'un rugissement ${ }^{13}$ ) sont occupés par des champs vides et des silences (Alice s'est arrêtée elle-même). Le plus frappant est que ce qui connote la présence de l'animal n'est rien d'autre que l'absence la plus absolue de l'humain et de ses traces (sa figuration et les sons qui lui sont propres). Voilà le guet dans son état idéal : Irena est aux aguets d'autant plus qu'on ne la voit pas, tout en sachant qu'elle est quelque part au bord du cadre. Oserions-nous dire qu'elle est en fait au seuil du cadre? Mais où? Et surtout, ce qui guette au seuil du cadre, est-ce Irena sous sa forme reconnaissable? Quelle violence corporelle est-elle en train de subir? Le vide, et rien que le vide, en est la seule réponse.

À vrai dire, la seule affirmation apparaissant comme légitime veut que l'humain se soit égaré et que son destin, quel qu'il soit, 
ne soit pas donné à l'œil. N'est-il pas significatif qu'un nouveau déchaînement associatif naisse en ce moment et autour de cette double absence? Plan subjectif d'Alice (plan 26) : contreplongée d'un arbuste agité par le vent.

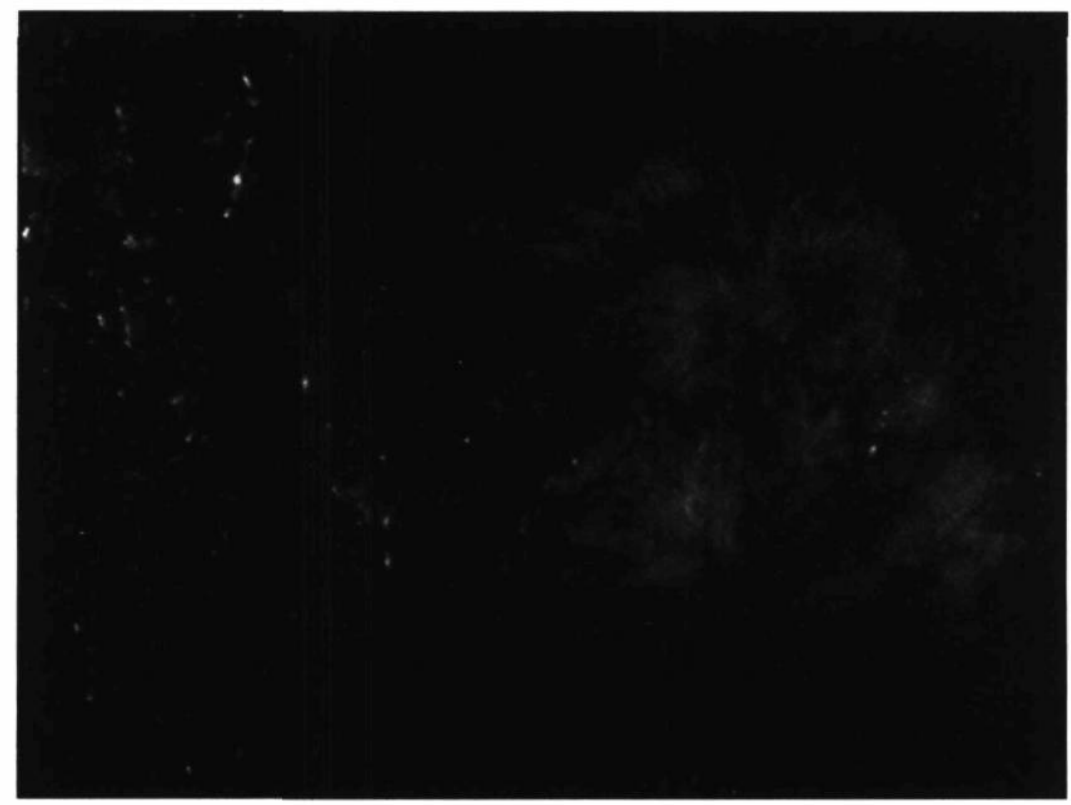

Un nouveau champ vide hanté par quelque chose qui produit un effet bien visible, tout en restant extérieur au champ. Lorsque Alice quitte la scène, l'énonciation nous conduit à travers une logique associative cryptique, voire incompréhensible à moins qu'on ne la lise en surimpression:

Plan 28: Champ vide des arbustes agités par le vent (recréation du point de vue antérieur d'Alice).

Plan 29: Plan serré de la panthère dans sa cage (dès l'intérieur) (photogramme 21). Ce plan couvre figurativement ce qui restait vide dans le précédent. Ce qui revient à dire qu'il représente la cause de l'effet antérieur (le mouvement). Ajoutons que ce plan comble également d'un point de vue sonore le vide de l'autre (rugissement de la panthère).

Plan 30 : Léopard dans sa cage vu de l'extérieur.

Plan 31 : Plan général d'un troupeau qui bêle. Quelques agneaux restent sur le sol. Morts. Voilà à nouveau 
un effet métonymique de la panthère, mais faut-il préciser que cette lecture n'est aucunement obligatoire?

Plan 32: Plongée sur des traces vraisemblablement félines illuminées par la lanterne du berger. Ce plan rime avec les pas qui ont pris tant d'importance préalablement.

Plan 33: Plan entier du berger (comme la fin de 31) qui siffle.

Plan 34 : Glissement d'un travelling latéral de gauche à droite illuminant des traces semblables aux précédentes, mais à présent sur le pavé. Or, cette fois-ci, la lumière n'est plus guidée par le berger; il s'agit évidemment d'un geste énonciatif fort marqué. Un nouveau déplacement: plus de terre, mais du pavé. Une récurrence, donc, et une transformation. Le sifflet - celui du berger? - sonne à nouveau. La source sonore n'est pas visible et, par conséquent, la distance qui nous sépare d'elle, ainsi que son identité même, sont impossibles à déterminer. Nouvelle transformation: les traces acquièrent peu à peu un aspect humain, celles de souliers à talon. Soudain, on a l'impression de suivre quelqu'un. Peut-être s'agit-il de l'animal en son moment de transformation inverse (de récupération de l'aspect humain) ? En tout cas, tout renforce la sensation que le noyau dramatique est en train de se jouer, une fois de plus, sur les bords du cadre. Et, en réalité, c'est de ce côté-là que l'on écoute des pas; les pas, en fait, qui s'étaient égarés de la bande-son quelques plans auparavant.

Plan 35: En faisant un raccord de direction avec le mouvement d'appareil précédent, Irena entre dans le champ par la gauche en plan moyen. Elle semble en extase et porte son mouchoir sur la bouche. Limpression immédiate suppose qu'il s'agit du même plan puisqu'une continuité parfaite fournit un raccord. Néanmoins, si nous réfléchissions un peu, il nous serait impossible de décider quel rapport spatial ont entre eux ces derniers plans. Y a-t-il eu un déplacement? S'agit-il, par contre, d'espaces contigus?

Il vaudrait la peine d'examiner de plus près cette logique discursive, qui pousse l'évanouissement de l'humain - interprété par le spectateur, d'après tous les indices, comme la manifestation d'une métamorphose qui a produit - dans les limites du 


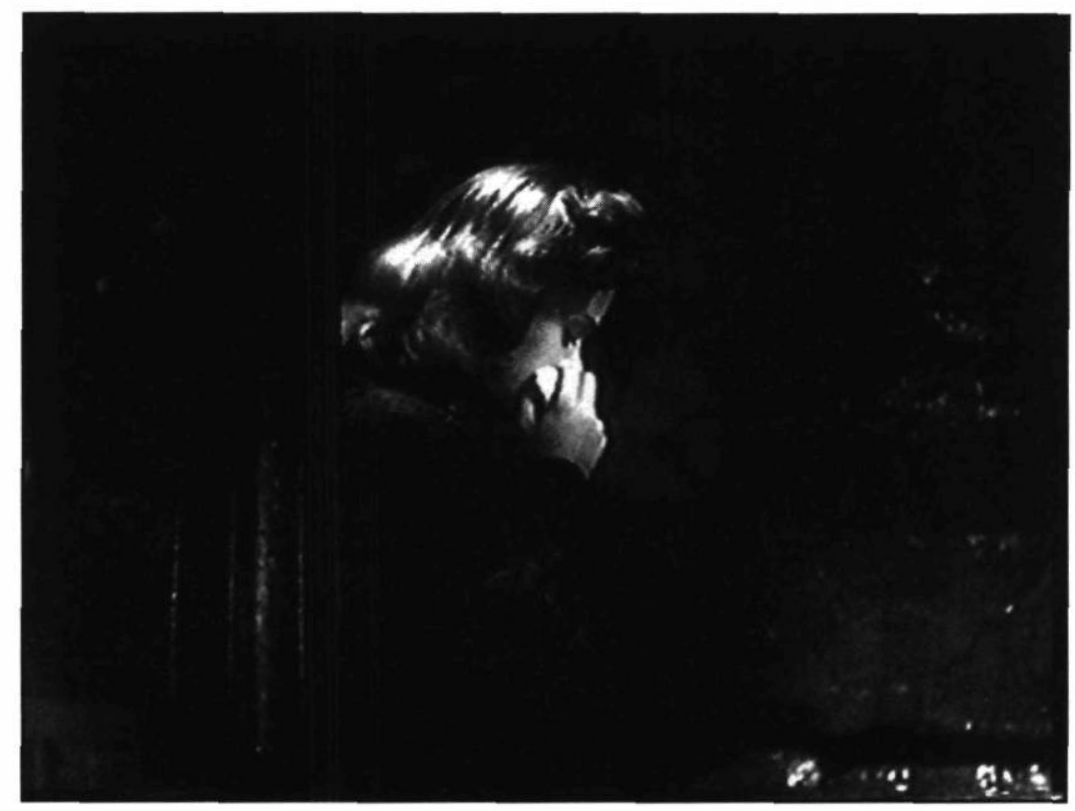

cadre, tout près du regard, mais légèrement déviée par rapport à lui. Elle lui échappe, si l'on peut dire. Une chaîne métonymique prend la relève aussitôt, elle permet de lire en surimpression, c'est-à-dire d'un point de vue aussi métonymique que métaphorique, la métamorphose. Précisons-le davantage, ce n'est qu'en lisant ainsi que les signifiants isolés acquièrent un sens. Le premier et le second sifflet proviennent-ils de la même source? Le zoo et le parc sont-ils près l'un de l'autre? Ou, encore, la terre sur laquelle le troupeau marche est-elle près du pavé sur lequel Irena se promène? Les traces marquées sur le pavé sont-elles contiguës à l'entrée dans le champ d'Irena? Autant de fractures qui ont été organisées selon une logique métonymique, mais qui - faut-il le dire? - comblent métaphoriquement le lieu d'une absence. L'attitude de l'énonciation rend impossible une cohérence non associative. Cependant, nous ne sommes aucunement autorisés à interpréter l'association comme si elle était une assertion. Nous ne sommes autorisés qu'à suivre la chaîne et à rendre compte des ruptures. 


\section{Pour (ne pas) conclure}

Nous aimerions nous poser une dernière question: à quoi bon la conversion de Cat People en film culte de la part des historiens? Comme nous l'avons mentionné précédemment, le cinéma des derniers temps offre à notre œil un présent empoisonné: toutes les métamorphoses qui se produisent (et elles sont très nombreuses) ont lieu dans le champ, sans qu'un seul détail de la violence et des déformations subies par le corps humain ne soit perdu ${ }^{14}$. Autrement dit, le cinéma de terreur (et il faut ajouter qu'il a contaminé d'autres genres à cet égard) s'efforce de faire rentrer dans le champ ce qui restait sur les bords du cadre dans Cat People; ou encore, de donner à l'œil ce qui était autrefois dissimulé à l'intérieur du récit. Nous avons affaire aujourd'hui à une attitude de la représentation qui se refuse à laisser quoi que ce soit en dehors de la vision, mi-caché à l'œil ${ }^{15}$. Cette supposée plénitude du regard, nous la retrouvons de façon exemplaire et sous son mode postmoderne dans ce qu'on nomme les Splatter Movies.

Faudrait-il reformuler la question que l'on vient de poser? Pourquoi les films de Val Lewton-Jacques Tourneur sont-ils devenus des films cultes malgré leur style archaïque par rapport à l'actualité cinématographique? La nostalgie est-elle une explication? Tout cela semble confirmer, une fois de plus, cette maxime de la postmodernité qui soutient non seulement que tout est possible, mais aussi que tout est possible simultanément.

\section{Université de Valence, Espagne}

\section{NOTES}

1 Ce qui a été établi et documenté par Joel Siegel dans Val Lewton: The Reality of Terror (New York: Viking Press, 1973).

2 Voilà une preuve a contrario de la cohésion du groupe: le fait que la photographie de ces trois films présente des traits communs, malgré avoir été conçue par trois spécialistes (Nicholas Musuraca pour Cat People, Roy Hunt pour I Walked with a Zombie et Robert de Grasse pour The Leopard Man).

3 Jacques Tourneur, consulté tout au long de sa vie à propos du fantastique, n'a cessé de répéter à quel point il détestait ces films de vampires et créations imaginaires de facture gothique. 
4 Tom Gunning dans «Like unto a Leopard: Figurative Discourse in Cat People and Todorov's The Fantastic" (Wide Angle, vol. 10, $\mathrm{n}^{\circ} 3,1989$ ) aborde la particularité du fantastique dans Cat People à partir de la construction discursive biblique "Like Unto a Panther " qui sert à désigner une identité floue, incertaine.

5 Bernard Eisenschitz développe cette idée dans "Six films produits par Val Lewton", dans Raymond Bellour (direction), Le Cinéma américain. Analyses de films, vol. 2 (Paris: Flammarion, 1980, p. 49).

6 Il est possible de consulter à ce sujet le livre de J. P. Telotte, Dreams of Darkness. Fantasy and the Films of Val Lewton (Urbana / Chicago: University of lllinois Press, 1985), notamment le chapitre premier.

7 Voici le sujet de notre livre en préparation, Despedazar un cuerpo. El cine de terror desde Psicosis.

8 Jesús González Requena a parlé de cet œil comme celui qui jouit en déchirant le corps féminin ("Desplazando la mirada. Hitchcock vs Griffith ", Contracampo, n 38, 1985 , p. 32).

9 C'est dans ce sens d'offre à l'œil que John McCarty essaie de définir les Splatter Movies: la mutilation serait ainsi le seul message (Splatter Movies. Breaking the Last Taboo of the Screen, New York: St-Martin's Press, 1984, p. 1).

10 Jacques Tourneur racconta plus tard que l'ombre qui apparaît dans la scène de la piscine fut obtenue à partir de son poing et fut tourné à l'aide d'un écran diffuseur.

11 Il s'agit ici peut-être de la seule apparition de la panthère au-delà des ombres. Voilà l'expression figurative de l'exigence de la RKO : il fallait devenir plus compétitif avec l'Universal et l'on ajouta quelques mètres représentant la panthère menaçante. Voir l'ouvrage de Telotte, Dreams of Darkness. Fantasy and the Films of Val Lewton (Urbana / Chicago: University of Illinois Press, 1985, p. 21).

12 En effet, préalablement, les deux femmes s'avançaient en raccordant leurs mouvements, malgré leur distance. Ce qui équivaut à dire qu'elles allaient vers leur rencontre: l'une vêtue de blanc, l'autre avec un manteau noir.

13 Il s'agit ici de ce que Telotte appelle bus technique.

14 Le remake de Cat People par Paul Schrader (1982) en constitue un exemple fort représentatif. Mais il y en a des millions dans les films de David Cronenberg, Lamberto Bava, Daniel Attias, Kevin S. Tenney, etc.

15 Jesús G. Requena a longuement étudié ce phénomène du point de vue psychanalytique en le qualifiant de "menace du réel" (El espectáculo informativo o la amenaza de lo real, Madrid: Akal, 1989).

\section{OUVRAGES CITÉS}

Doane, Mary Ann. The Desire to Desire. The Woman's Film to the 40s. Bloomington: Indiana University Press, 1987.

Gunning, Tom. "Like unto a Leopard: Figurative Discourse in Cat People and Todorov's The Fantasticn. Wide Angle, vol. 10, n 3, 1989.

Jacobs, Lea. "The B Film and the Problem of Cultural Distinction". Screen, vol. 33, $n^{\circ} 1,1992$.

Lowins, Evelyne. «Tourneur, Robson, Wise». Cinéma d'aujourd'hui, n 3, 1975. 